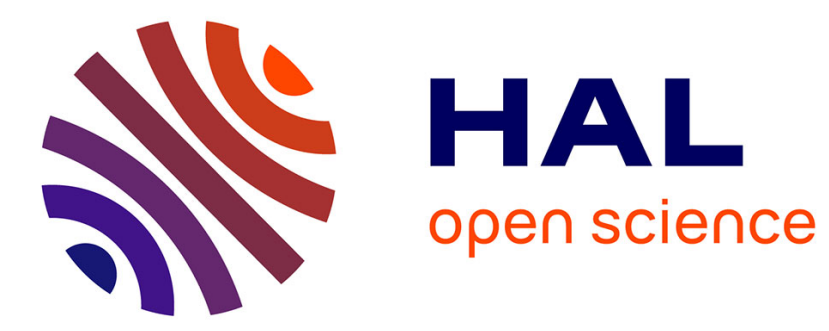

\title{
Geometry of the superior colliculus mapping and efficient oculomotor computation
}

Nicolas Tabareau, Daniel Bennequin, Alain Berthoz, Jean-Jacques Slotine, Benoît Girard

\section{- To cite this version:}

Nicolas Tabareau, Daniel Bennequin, Alain Berthoz, Jean-Jacques Slotine, Benoît Girard. Geometry of the superior colliculus mapping and efficient oculomotor computation. Biological Cybernetics (Modeling), 2007, 97 (4), pp.279-292. 10.1007/s00422-007-0172-2 . hal-00178144

\author{
HAL Id: hal-00178144 \\ https://hal.science/hal-00178144
}

Submitted on 10 Oct 2007

HAL is a multi-disciplinary open access archive for the deposit and dissemination of scientific research documents, whether they are published or not. The documents may come from teaching and research institutions in France or abroad, or from public or private research centers.
L'archive ouverte pluridisciplinaire HAL, est destinée au dépôt et à la diffusion de documents scientifiques de niveau recherche, publiés ou non, émanant des établissements d'enseignement et de recherche français ou étrangers, des laboratoires publics ou privés. 


\section{Nicolas Tabareau • Daniel Bennequin · Alain Berthoz • Jean-Jacques Slotine • Benoît Girard \\ Geometry of the superior colliculus mapping and efficient oculomotor computation}

\begin{abstract}
Numerous brain regions encode variables using spatial distribution of activity in neuronal maps. Their specific geometry is usually explained by sensory considerations only. We provide here, for the first time, a theory involving the motor function of the superior colliculus to explain the geometry of its maps. We use six hypotheses in accordance with neurobiology to show that linear and logarithmic mappings are the only ones compatible with the generation of saccadic motor command. This mathematical proof gives a global coherence to the neurobiological studies on which it is based. Moreover, a new solution to the problem of saccades involving both colliculi is proposed. Comparative simulations show that it is more precise than the classical one.
\end{abstract}

The original publication is available at www.springerlink.com

DOI 10.1007/s00422-007-0172-2

Keywords Saccades · Superior Colliculus · SpatioTemporal Transformation · Computational Model

\section{Introduction}

Successful goal-oriented movements rely on the ability to $\downarrow$ transform sensory inputs signaling the position of the target into appropriate motor commands. This transformation re$\infty$ quires representation changes from the sensory input space $\checkmark$ to the motor output space. Even in the case of visually guided

This work is partly supported by the EU within the NEUROBOTICS integrated Project (The fusion of NEUROscience and roBOTICS, FP6IST-FET-2003no. 001917).

N. Tabareau · A. Berthoz $\cdot$ B. Girard

Laboratoire de Physiologie de la Perception et de l'Action, UMR 7152, CNRS - Collège de France, Paris, France.

Tel.: +33-1-44-27-13-91, Fax: +33-1-44-27-13-82 E-mail: benoit.girard@college-de-france.fr

D. Bennequin

Institut de Mathématiques de Jussieu, UMR 7586, Paris, France.

J.-J. Slotine

Nonlinear Systems Laboratory, Massachusetts Institute of Technology, Cambridge, Massachusetts, USA. ocular saccades, a relatively simple sensorimotor transformation, the details of this computation are still debated.

The generation of ocular saccades greatly involves the superior colliculus (SC) (the tectum in non-mammalian vertebrates). The SC is a layered structure located in the midbrain, which receives multisensory input and accordingly generates changes in gaze orientation. It drives, in particular, the reticular formation nuclei which contain the ocular saccade motoneurons (the saccade burst generators, SBG). In the SC, the sensory inputs and the corresponding output commands are represented on retinotopic neuronal maps. Each colliculus encodes the information corresponding to the contralateral visual hemifield. A specific logarithmic deformation on the amplitude axis of this mapping was found in cats (McIlwain 1976, 1983) as well as in monkeys (Robinson 1972; Ottes et al. 1986) (see Fig. 11), whereas the mapping seems to simply be linear in the other studied species (rats (Siminoff et al. 1966), goldfish (Herrero et al. 1998), for instance). These mappings are usually explained by a reasoning based on sensory considerations: if the projections from the retina to the SC are one-to-one and if the density of cells in the collicular maps is constant, then the absence or existence of a fovea induces linear or logarithmic mappings. We propose here an alternative approach linking these mappings with the saccadic sensorimotor transformation process.

This sensorimotor process involves the activation of a large population of cells in the motor map. This activation is centered around the position corresponding to the coordinates of the target of the saccade in the visual field (see upper part of Fig 11). The SBG are composed of four circuits, respectively producing the rightward, leftward, upward and downward rotations. At this level, the movements are encoded by bursts of activity representing the vectorial components of the desired rotation (see lower part of Fig 1). The transformation from the SC distributed spatial code into the SBGs Cartesian temporal code is called the spatio-temporal transformation (or STT). In addition to the problem of solving the STT for one colliculus, a gluing problem -in the technical sense of differential geometry Hirsch (1976) - occurs when a vertical or quasi-vertical saccade is executed. In that case, the population activity is shared on both SC and 
the combination of these two activities drives the SBG. The exact location and shape of this distributed activity, and the possible role of the commissural SC projections in the coordination of the two SC, are unknown.

The first model of the STT, proposed by van Gisbergen et al. (van Gisbergen et al, 1987), stated that it could be performed by a simple weighted sum of the activity of the SC neurons, transmitted to the SBG. This scheme has been reproduced in many early $\mathrm{SC}$ models (refer to (Girard and Berthoz 2005) for a review of SC and SBG models). It assumed that the spatial shape of the activation on the SC map is stereotyped, which could be ensured by lateral connections inside the map. This model had some limitations: it did not simulate correctly the effects of simultaneous multiple site activation (saccade on the average position), of varying levels of peak activity (saccades are accurate for various peak levels of activity), and of inactivation of parts of the SC (the inactivated region "repels" saccades). The saccade averaging concern was solved in a model including lateral inhibitions within the colliculus (van Opstal and van Gisbergen 1989). However, the most important limitation is that the dynamics of appearance and disappearance of the SC activity, implying varying levels of activity, wasn't considered, namely, it did not take time into account.

In competing models, it was proposed that the output of the $\mathrm{SC}$ is normalized by a weighted averaging of its activity. This allowed the generation of correct saccades with varying levels of activity, and simulated the effects of multiple target averaging and of inactivation of collicular regions Lee et al. 1988. However, as noted by Groh Groh 2001, the division computation is critical in such a model, as it has to be carried out by a single neuron (this computation cannot be broken up among a population) and should be precise on a large range of values, which is physiologically unrealistic.

Recent experimental studies shed light on the dynamics of the saccade generation process, showing that the number of spikes produced by the whole population of SC burst neurons during saccades of different amplitudes is constant (Anderson et al. 1998; Goossens and van Opsta 2006). Moreover, it was also shown that for a given saccade, individual SC neurons always produce the same number of spikes, even in case of various kinds of perturbations: saccades interrupted by fixation zone stimulation (Munoz et al. 1996), saccades slowed by muscimol injection in omnipause neurons region (Soetedjo et al. 2000), and saccades perturbed by eye blinks (Goossens and van Opstal 2000, 2006). This strengthens recent STT models (Groh 2001; Goossens and van Opstal 2006), which take time into account, and where it is assumed that an inhibitory mechanism keeps the number of spikes constant, avoiding the need for normalization. The "dynamic vector summation" model, proposed by Goossens and van Opstal (Goossens and van Opstal 2006), implements this mechanism in a manner very similar to the Groh (Groh $2001)$ "summation with saturation" proposal: a population of neurons sums up the number of spikes emitted by the SC and inhibits the SC output when a fixed threshold is reached. These models exhibit satisfactory behaviors in case of mul-

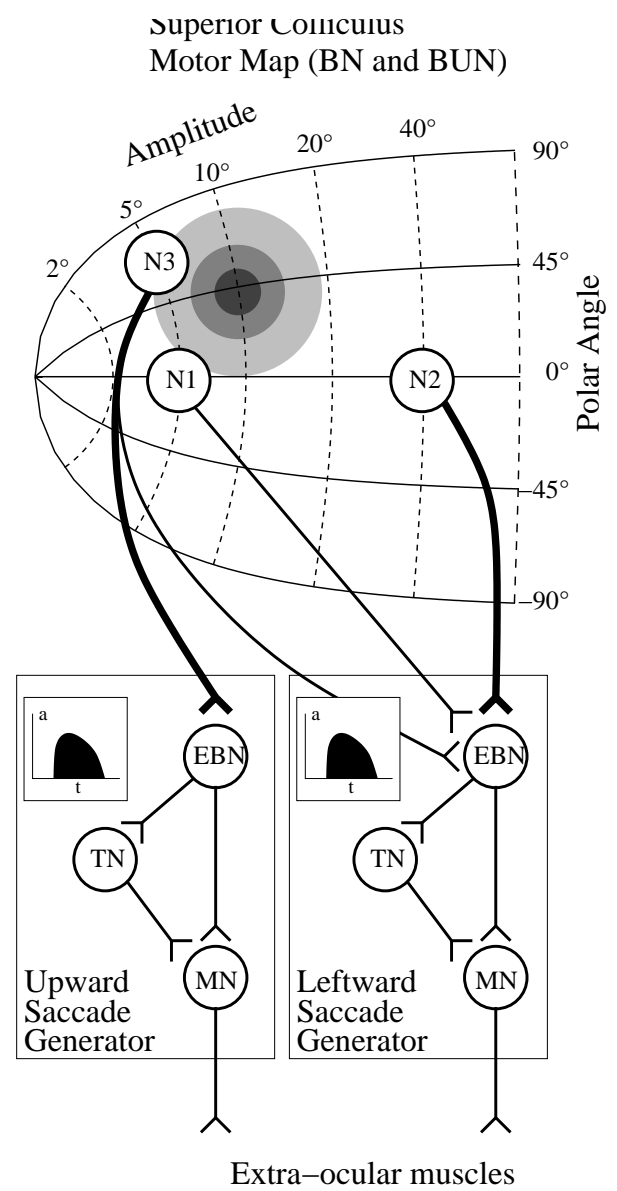

Fig. 1 Spatio-temporal transformation from the superior colliculus motor layers to the saccade generators. BN: burst neurons; BUN: buildup neurons; EBN: excitatory burst neurons; MN: motoneurons; TN: tonic neurons. Dashed lines on the SC map represent iso-amplitudes and full lines, iso-directions. Gray shading on the SC map represents the activity of the population of neurons coding for a $\left(R=10^{\circ}, \theta=\right.$ $45^{\circ}$ ) saccade. SBG are simplified: circuitry devoted to the triggering of saccades is omitted. Insets represent the temporal activity of the EBNs during the execution of the saccade. The transformation from spatial to temporal coding results from selective weighted projections from SC neurons to the SBGs (strength is represented by line width): neurons $\mathrm{N} 1$ and N2 project to the leftward SBG only, as they code for horizontal saccades, and the N2 projection is stronger as it codes for a saccade of larger amplitude; neuron N3 projects to both upward and leftward SBGs as it codes for a $\left(R=5^{\circ}, \theta=67.5^{\circ}\right)$ saccade.

tiple site activation, varying levels of peak activity and inactivation of parts of the SC.

Finally, the gluing problem was addressed in the van Gisbergen et al. study (van Gisbergen et al. 1987). Their proposal is based on a geometrical construction which only partially uses the logarithmic mapping and systematically generates inaccurate saccades. It is also this form of gluing which was used in the Goossens and van Opstal model Goossens and van Opsta 2006.

In this work, we prove that, using a set of six hypotheses based on known neurobiology of the SC and of the SBG and fully compatible with the last two STT models Groh 2001; Goossens and van Opstal 2006, the neural implementation 
of the STT is tightly linked with the geometry of the collicular mapping: it is necessarily linear or complex logarithmic. Moreover, we propose a new gluing scheme which extends these STT models to both SC, generates accurate saccades, and is compatible with the requirements of our proof.

\section{Results}

The quantitative description of the monkey's collicular mapping proposed by Ottes et al. Ottes et al. 1986) can be reformulated as a complex logarithm (refer to appendix 4.1 for more detailed considerations about quantitative description of the collicular mapping). This transformation from retinotopic Cartesian coordinates $(\alpha, \beta$, resp. azimuth and elevation) into coordinates on the SC surface $(X, Y$, in millimeters) is expressed as follows:

$$
\frac{X}{B_{X}}+i \frac{Y}{B_{Y}}=\ln \left(\frac{z+A}{A}\right), \text { with } z=\alpha+i \beta
$$

The values of parameters $A, B_{X}$ and $B_{Y}$ for the monkey have been experimentally estimated. Concerning the cat, the mapping is in accordance with such a description McIlwain 1976), but the parameters' values haven't been estimated. For animals having a linear mapping, the following formulation can be simply used:

$$
\frac{X}{b_{X}}+i \frac{Y}{b_{Y}}=z
$$

2.1 The need for a linear or complex logarithmic mapping.

Our first result is a mathematical proof (detailed in appendix 4.2) that the complex logarithmic or linear mappings (as defined by eqns. 1 1 and 2) are the only appropriate ones. Interestingly, these classes of mappings are conformal (as the functions are holomorphic) although it is not required by the hypotheses on which the proof is based. These hypotheses are based on the formalization of six known biological properties of the STT (their precise mathematical formulation is given in Appendix):

Weighted sum. The outputs of the SC fed to the horizontal and vertical saccade generators (SBG) are generated by weighted sums of the activity of the SC motor cells.

Glued colliculi. The two colliculi are connected with each other so that they form only one abstract mapping on the whole plane $\mathbb{R}^{2}$.

Invariant integral. For each motor cell, the number of spikes emitted during a whole saccade burst (without those corresponding to the eventual preceding build-up activity) depends only on its location with respect to the $(X, Y)$ coordinates of the saccade on the collicular surface.

Linearity. The total command sent from the SC to the SBG is a linear function of $z_{0}$, the Cartesian coordinates of the saccade to be generated.
Smooth mapping. The collicular mapping is continuously differentiable. $(X, Y)=(0,0)$ corresponds to $z=0$, and the visual horizontal and vertical axes are aligned with the $X$ and $Y$ axes in 0 .

Similarity. For any continuous population activity respecting the invariant integral hypothesis, the projection weights from the SC to the SBG is a similarity ${ }^{1}$ with regards to the saccade coordinates expressed in azimuth and elevation (the retinotopic Cartesian coordinates).

This similarity hypothesis is the less intuitive of our six hypotheses as it does not seem to have any functional justification. However, if it is assumed that the mapping on each side is either linear or logarithmic (a constraint which can be due to the appearance of a fovea), we prove in appendix 4.4 that for any activity with gaussian invariant integral, the only system of projection weights from SC to SBG (with a moderate growth), which produces a correct saccade (under the assumption of linearity), is a similarity. We also prove in this appendix that, given any activity, there is no deformation (with support on one SC) of the projection weights, except similarities, generating correct saccades. In particular this implies that the set of similarities is the only class of projection weights from SC to SBG, which can be adapted to every activity and which is stable under affine re-mapping (or modulation).

This analysis provides as a corollary an expression for the projection weights of the SC to the SBGs in the logarithmic case (it seems to be a folklore result although it never appeared in the literature). Using the equation of the logarithmic mapping, the projections from the superior colliculus to the brainstem can be analytically expressed. In the special case where the coefficients $a$ and $b$ of the hypothesized similarity are just real numbers, we obtain:

$$
\begin{aligned}
& w_{\alpha}=a A\left(\exp \left(\frac{X}{B_{X}}\right) \cos \left(\frac{Y}{B_{Y}}\right)-1\right)+b \\
& w_{\beta}=a A \exp \left(\frac{X}{B_{X}}\right) \sin \left(\frac{Y}{B_{Y}}\right)
\end{aligned}
$$

A graphical representation of this analytic formulation is given in Fig. 5. upper part, using the monkey's parameters.

The fact that the total activation of one neuron on the superior colliculus during the saccade depends only on its position with regard to the point coding the saccade on the collicular surface is fundamental in inducing a logarithmic mapping. However, if we assume that the mapping is logarithmic and that interindividual differences in the mapping parameters $\left(A, B_{X}, B_{Y}\right)$ within one species exist, we can derive the invariant integral hypothesis from the five others (proof in appendix 4.5).

In the course of the mathematical proof, a parameter which triggers the shape of the mapping appears: if it is null, then the mapping is linear, otherwise, it is complex logarithmic. The transition from linear to complex logarithmic is smoothly obtained by a continuous variation of this parameter. This means that during evolution, a transition from

\footnotetext{
1 a similarity is a transformation that preserves ratios of distances.
} 
a linear to a complex logarithmic mapping could have happened without any need for changing the neural structures in charge of computing the STT.

\subsection{The motor gluing of colliculi.}

We assumed in our glued colliculi hypothesis that the two colliculi are connected so that the combined activity of their motor layers can be considered as a single abstract mapping on the whole plane $\mathbb{R}^{2}$. To solve the gluing problem in the linear mapping case, it is sufficient to put a bump of activity in each SC at the correct position, to truncate it to keep the part within the correct visual hemifield only and then to use the sum of the activity of both colliculi to drive the SBG. However, in the case of complex logarithmic mapping, a similar approach produces systematic errors (see Simulation section below).

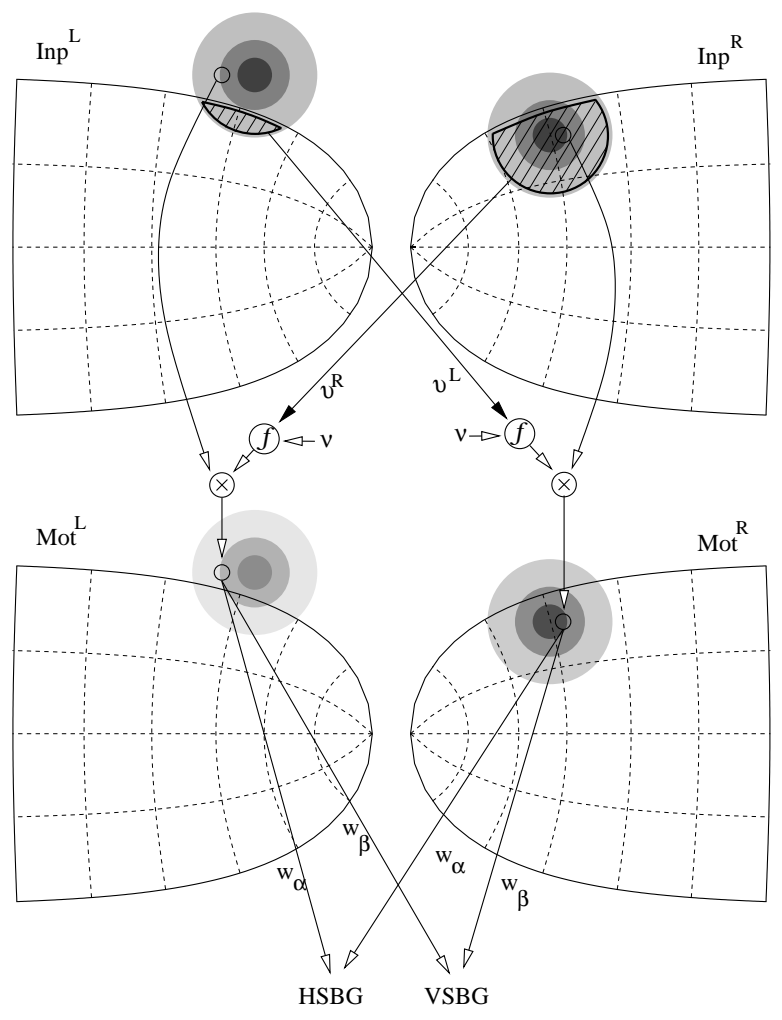

Fig. 2 Gluing method. A single target in the left hemifield but close to the vertical elicits activity in the input layers of both colliculi ( $\operatorname{In} p^{R}$ and $\left.I n p^{L}\right)$. In the motor layers $\left(M o t^{R}\right.$ or $\left.M o t^{L}\right)$ this activity is inversely modulated by the area of the contralateral activity within the boundary of its visual hemifield (hatched area, noted $v^{R}$ and $v^{L}$ ). Note that $\nu$ is the sum of the activity of the whole shaded areas. In the motor layer, activity is thus much stronger in the right colliculus (coding the left hemifield) than in the left one. For a target further away from the vertical, there would be no activity left in the left motor layer. This distributed motor activity is the abstract $\mathbb{R}^{2}$ mapping assumed by the second property of our first proof, which can then be weighted, summed and sent to the horizontal and vertical SBG.
To solve this problem, we propose another approach. It consists in progressively shifting from an activity shared by both colliculi to an activity contained by a single representation, using a modulation accounting for the closeness to the vertical axis. In this scheme, an input layer $\left(\operatorname{In} p^{R}\right.$ or $\left.\operatorname{In} p^{L}\right)$ receives activation from visual sources, independently from the activity in the contralateral visual layer (Fig. 2, upper part). These layers project to the motor layers $\left(M o t^{R}\right.$ and $M o t^{L}$ ) of the ipsilateral and contralateral colliculi (Fig. 2, lower part). The ipsilateral projections are one-to-one connections: each visual neuron projects to its homologue in the motor layer. These projections are however modulated by the relative part of the activity of the contralateral input layer within the boundary of its visual hemifield. This modulation is a monotone increasing function $f$ of the subtraction of the sum of the activity within the boundary $\left(v^{R}\right.$ and $\left.v^{L}\right)$ to the sum of the activity in the whole map $\nu$. The addition of a control mechanism ensuring the invariant integral property on the two motor maps ensures that the following holds for every saccade:

$$
\left\{\begin{array}{l}
\int_{t} \operatorname{Mot}_{S_{0}^{L}}^{L}\left(S^{L}, t\right)=\chi\left(S_{0}^{L}\right) \cdot \int_{t} \operatorname{In} p_{S_{0}^{L}}^{L}\left(S^{L}, t\right) \\
\int_{t} \operatorname{Mot}_{S_{0}^{R}}^{R}\left(S^{R}, t\right)=\eta\left(S_{0}^{R}\right) \cdot \int_{t} \operatorname{Inp}_{S_{0}^{R}}^{R}\left(S^{R}, t\right)
\end{array}\right.
$$

where saccade coordinates on the left (resp. right) SC are noted $S_{0}^{L}$ (resp. $S_{0}^{R}$ ). The positive functions $\chi, \eta$ are the result of the integrated commissural modulation and satisfy $\chi\left(S_{0}^{L}\right)+\eta\left(S_{0}^{R}\right)=1$ for all saccade. This constraint ensures that the sum of the activity on both colliculi behaves exactly as a single activity on an abstract map (a complete description of the scheme is given in Appendix).

\subsection{Simulation.}

To assess the accuracy of this gluing scheme, and also to compare it with the van Gisbergen et al. proposal (van Gisbergen et al. 1987), we built a simple computational model of the SC and SBG based on the Groh architecture for STT Groh 2001) (see Fig. ). This model is made of rate-coding leaky-integrator neurons. Each SC contains two $90 \times 90$ neuron maps, a visual input one and a motor one, respecting the monkey mapping equation from Ottes et al. Ottes et al. 1986). The activity generated by a target is a 2D Gaussian $(\sigma=0.5 \mathrm{~mm})$ centered on the target coordinates expressed in the collicular mapping. The activity of the motor map is controlled by a summation with saturation architecture. The SBGs' implementation is minimal, they contain no feedback loop, and are made of inhibitory and excitatory burst neurons receiving the output of the SC motor layer, of tonic neurons integrating the burst neurons activity and of motoneurons summing the burst and tonic neuron outputs. The eye plant is simulated by the standard second order differential equation model, linking eye rotation and motoneurons firing rate. Details of the model are given in appendix 4.7 . 

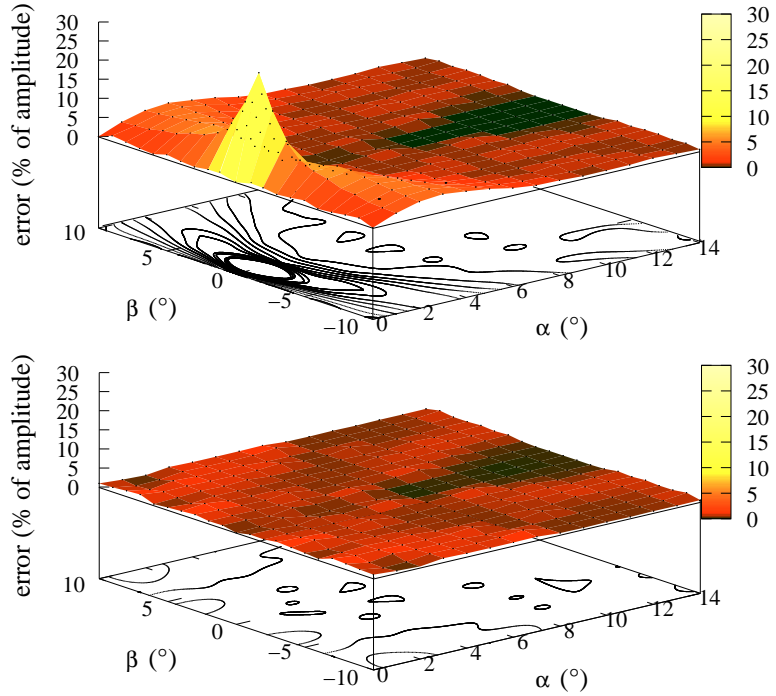

Fig. 3 Saccades endpoint error maps. 3D representation of the ratio of the distance between desired and generated saccade endpoints and the amplitude of the desired saccade, for saccades generated by the van Gisbergen et al. gluing scheme (top) and our proposal (bottom). Note that the van Gisbergen et al. proposal generates systematic errors close to the vertical, the result of an incorrect gluing. $\alpha$ : azimuth; $\beta$ : elevation.

In the van Gisbergen et al. van Gisbergen et al, 1987) proposal for gluing, when a saccade is so close to the vertical that the activity on the SC crosses the $90^{\circ}$ or $-90^{\circ}$ iso-direction curves, a second bump of activity is placed in the other SC, and the two bumps are truncated to keep the part within the preferred hemifield of each SC only. However, rather than using the mapping equation (1), they use a ad-hoc geometrical construction to place the second bump. This construction generates systematic errors for saccades close to the vertical (see their Fig. 4). In our simulation, we tested their truncation gluing scheme, but positioned the second bump according to the mapping equations. Even with this enhancement, relatively large systematic saccades errors are generated: the upper part of Fig. 3 shows the error (measured as the distance between desired and effective saccade endpoint divided by the desired saccade amplitude) for saccades generated over the $\left[0^{\circ}, 14^{\circ}\right]$ horizontal interval and the $\left[10^{\circ},-10^{\circ}\right]$ vertical interval, with a $1^{\circ}$ increment. This error, which reaches more than $27 \%$ for the $\left(1^{\circ}, 0^{\circ}\right)$ saccade, is around $5 \%$ in the vertical region, where gluing occurs.

The implementation of our model produces errors that are comparatively much lower (less than $1.5 \%$, lower part of Fig. B). These errors are caused by integration approximations when numerically solving the model's differential equations and by the coarse discretization of the SC, rather than by an approximate gluing.

\section{Discussion}

We showed that collicular mapping has to be either linear or logarithmic in order to control the SBG correctly, assuming six basic properties of the spatio-temporal transformation. This result also shows that a continuous transition from the linear to the logarithmic mapping can be made, affecting neither the neural substrate nor the underlying computations generating saccadic movements. In an evolutionary perspective, it suggests that the appearance of a fovea and the corresponding modification of the mapping of the visual areas could have happened in a progressive manner without requiring any modification of the final stages of the saccadic circuitry.

A hypothesis of this first result is that the two colliculi have to be combined so as to be equivalent to a single abstract mapping of the whole visual field. We thus proposed a new gluing scheme which generates saccades of the correct size and predicts the role and structure of the commissural projections in charge of driving this motor gluing.

\subsection{The six basic properties.}

We first discuss the neurobiological relevance of the six properties on which we based our proof, for the monkey and the cat.

The weighted sum property corresponds to the simplest way to transmit the activity of a population of SC neurons to the $\mathrm{SBG}$, as no additional circuitry is needed between SC motor cells and SBG bursters in order to, for example, select the most active neuron only. Moreover, relying on such a population coding is more resilient to noise in neural activity. This hypothesis has received support from both experimental (Sparks et al. 1976: Moschovakis et al, 1998) and modeling (van Gisbergen et al. 1987; Badler and Kellet 2002) studies.

The invariant integral property states that the shape of the activity on the SC map $\mathcal{A}$ does not have to be perfectly invariant in space and time, as long as the activity of each cell integrated over saccadic signal duration (ie. number of spikes emitted during the saccadic burst) depends only on its location with regards to the point on the SC surface coding for the saccade metrics. This hypothesis is weaker than the invariant Gaussian used in numerous models, it avoids putting too much constraint on the precise tuning of the activity profiles of the SC neurons (as depicted in Fig. (4). Not demanding temporal stereotypy allows the duration of a saccade of a given metric to vary from one execution to another, for example because of varying peak levels of activity, as long as the integrated activity is constant.

As mentioned in introduction, a number of recent experimental studies Anderson et al. 1998; Munoz et al. 1996; Soetedjo et al. 2000\$ Goossens and van Opstal 2000, 2006) with monkeys show that the number of spikes emitted by a given SC neuron for a given saccade is constant, despite various types of perturbation. This fully supports our invariant 


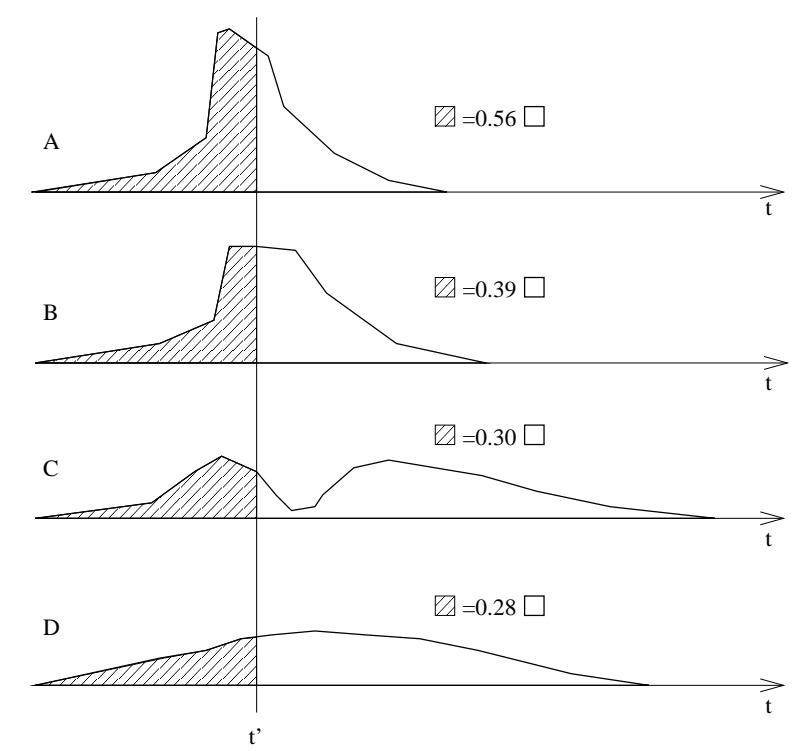

Fig. 4 Consequence of the invariant integral hypothesis for one SC neuron. A, B, C and D are schematic drawings of the activity of a given neuron of the $\mathrm{SC}$, for a given saccade metric. While A represents a normal saccade, B, C and D represent the activity of the neuron in perturbed saccades (like during stimulation of the fixation cells, muscimol injection in the OPNs, eye blink, etc.). In all cases, the integrated activity over the whole burst duration (the surface within the bold polygon) is constant, thus these activations are compatible with the invariant integral hypothesis. Note that, at a given moment $t^{\prime}$, the generated fraction of this activity (represented by the hatched surface) may vary.

integral hypothesis, at least for monkeys. We are not aware of similar results in cats that could shed a complementary light on our invariant integral hypothesis. Since SC neurons have quite different morphology and physiology in felines and primates (Grantyn and Moschovakis 2003), such studies are necessary to test the validity of the hypothesis in cats.

The linearity property states that the desired saccade amplitude has to linearly depend on the SC output. The burst neurons of the SBG, which receive this SC output and generate the phasic part of the motoneuron activity responsible for saccadic eye movement, exhibit an affine relationship between the number of spike they emit during a saccade and the amplitude of the saccade, in monkeys Kellen 1974; King and Fuchs 1979) as well as in cats (Kaneko et al. 1981; Yoshida et al.|1982). If the summed offsets of the affine functions of the burst neurons coding for two opposite directions are equal, then the fact that the SBG are controlled linearly holds. It happens that the SBG also receives input from the fastigial oculomotor region (FOR) of the deep cerebellar nuclei. It does not affect our proof, as we do not demand that the SBG input exclusively comes from the SC. However, it means that this affine relationship in the burst neurons is not the result of the SC influence only. Thus, the SC input signal might vary non-linearly with saccade amplitude, as the cerebellar input could compensate this non-linearity, so that the summed command remains linear. However, it was shown Iwamoto and Yoshida 2002 that in monkeys, an inactiva- tion of FOR results in a saccadic gain modification. This means that the suppression of the FOR input to the SBG generates saccadic movement whose amplitude still vary linearly with the amplitude of the desired saccade, proving that the collicular input to the SBG is also a linear command, whose gain is not 1 , and that has to be compensated by cerebellar input. Concerning cats, the effects are affecting either the gain or the offset for, respectively, contraversive and ipsiversive movements (Goffart and Pélisson 1998). However, this study was carried out head-free, similar head-fixed experiments would be necessary to validate or invalidate our hypothesis in cats.

The similarity property states that the projection weights from the SC to the SBG are a similarity of the saccade coordinates, expressed in the visual space. This unintuitive property was indeed derived from the evidence that in cats this projection is affine on the horizontal axis Moschovakis et al. 1998). However, neither the fact that the vertical projection is affine nor the fact that the whole projection function is a similarity, a subset of the affine functions, were proved in cats. Moreover, no result of that type is available for monkeys. However, as evoked in the results section, using the five other hypotheses and assuming that the mapping is either linear or complex logarithmic, we were able to prove that the weights respect the similarity hypothesis.

The appendix 4.6 of this paper contains a generalization of our results, showing that if we relax the similarity hypothesis by assuming affine projection only, three additional types of mappings become acceptable and all the resulting five mappings can be non-linearly twisted. Finding animals whose mapping corresponds to one of these three mappings would favor the affine hypothesis.

Note that this hypothesis is formulated so that similarity has to be true for any $\mathcal{A}$. Thus our result implies that with a complex logarithmic mapping, for any $\mathcal{A}$ function verifying the invariant integral property, the parameters $a$ and $b$ (defining the weights in eqn. 3) can be found so that a weighted sum of the activity of the SC neurons will generate accurate saccades. This means that the precise shape of $\mathcal{A}$ can change during lifetime and be different from one individual to another: an adaptive mechanism tuning $a$ and $b$ is sufficient to ensure correct operation of the system, there is no need for changing the mapping of the SC maps itself.

The hypothesis, that the similarity must be true for any $\mathcal{A}$ function verifying the property of invariant integral, is quite strong. However, our proof holds true even with restricted families of activations. For example, if the similarity has to be true for Gaussian functions with small perturbations of mean, we still obtain the two mappings.

Concerning the smooth mapping property, stating that the mapping function $\phi$ is continuous comes directly from the well known retinotopy of SC maps. Stating that its first derivative is also continuous means that the variation of the magnification factors on the maps are smooth, which is verified in all studied species. Finally, the $X$ and $Y$ axes used to describe the maps are chosen, by convention, so as to be aligned with the horizontal and vertical directions in 0 . 
The fact that these neurobiological properties and the known SC mappings can be combined together in a mathematical proof strengthens their coherence and reduces the concern of their individual uncertainties. Experimentally exploring the validity of these six properties in species other than cat and monkey, especially those having a linear mapping, could reveal whether our results can be generalized among vertebrates.

\subsection{SC to SBG projection weights.}
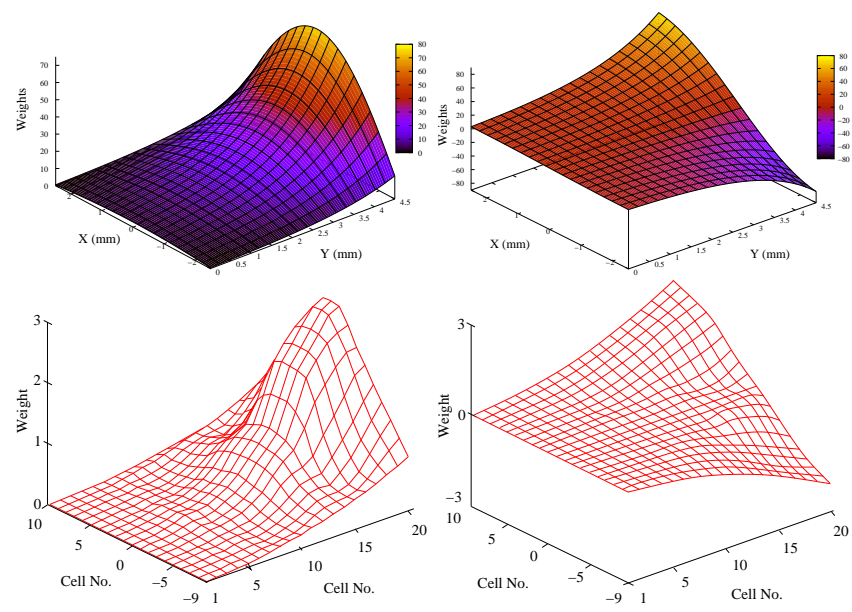

Fig. 5 Top: Plots of the weight from all the motor cells of the superior colliculus to the brainstem horizontal burst generator (left) and to the vertical burst generator (right) in monkeys. The values of these yeights were obtained by the exact equations described in text (eqn. (B)) and parameters specific to the monkey $\left(A=3^{\circ}, B_{X}=1.4 \mathrm{~mm}\right.$ and $B_{Y}=1.8 \mathrm{~mm}$ and $a=1 . b=0$. Bottom: Plots of the weights obtained by Arai et al. Arai et al. 1994 with a learning algorithm for a map covering from $0^{\circ}$ to $20^{\circ}$ in amplitude and from $-65^{\circ}$ to $65^{\circ}$ in direction.

As regards the projection from the superior colliculus to the saccade generator, we must say that to our delight similar profiles have been obtained by Arai et al. Arai et al. 1994) using a training procedure based on their model of the SC (see Fig. 5). It shows both that these weights can be obtained by learning and that our theoretical approach is corroborated by a more experimental one. Nevertheless, in another paper Arai et al. 1999, they obtained different profiles as they used a mixed velocity and position feedback to control SC activity, which transgresses our invariant integral hypothesis.

A few neurobiological studies tried to evaluate the weights of the connections from the SC to the SBG. The density of SC neurons projecting to the horizontal SBG in monkeys Grantyn et al.2002 have variation tendencies compatible with our results, at least for a range of saccades for which head movement are negligible. The technology available to estimate projection weights is however too limited yet to provide a full account of or to reject our result.

\subsection{Is there a STT?}

Optican (Optican2005) proposes that the sensorimotor transformation necessary to convert visual input into motor command doesn't need to be explicitly performed as a STT between the SC and the SBG. In his model, the SC gives only an initial directional drive to the saccadic system, while the cerebellum plays the major part, as it implicitly performs the transformation.

It can be reasonably assumed that the importance of the cerebellum has been neglected in previous modeling studies, as its role in the calibration of the system and in on-line adjustments of saccade trajectory is fundamental. It could indeed replace the reticular formation displacement integrator postulated by many former SBG models. Nevertheless, the available neurobiological data, that we use to build our proof, clearly shows that all the elements needed to perform a STT between the SCs and SBGs are present. We thus propose that a STT indeed occurs, with a gain different from 1 , and that the cerebellum constantly compensates for this difference.

\subsection{Commissural projections}

Commissural projections seem to exist at every level of the SC (Olivier et al. 1998), and many of them are probably used to solve various gluing problems, such as ensuring consistency of visual information in the superficial layers, or continuity of retinotopic working memory at the level of the quasi-visual cells. Our proposal uses a set of commissural projections to solve the gluing problem at the motor level, and thus makes predictions concerning these commissural projections only. Experimentally distinguishing these various types of commissural projection might be crucial for the understanding of their organization and roles.

\section{Appendix}

4.1 Coordinates on the SC layers and mapping formulation

The question of the nature of the coordinate system that should be used to describe the mapping on the collicular layers has to be raised. Indeed, the colliculus, and especially its superficial visual layers, are convex. The maps proposed in biological studies are obtained with various methods: projections on the Horsley-Clarke plane (Siminoff et al. 1966; Feldon et al. 1970; Robinson 1972; Dräger and Hugel 1976), empirical flattening of the surface by cutting (Rosa and Schmid 1994), or locally cylindrical coordinates (Knudsen 1982). None of these methods respects the curvature of the surface. Only Siminoff et al. (Siminoff et al, 1966) propose a correction -on two axes only rather than for the whole surfacethat takes the curvature into account.

Solving this question is beyond the scope of this paper, we however stress that our results concern the activity of the 
intermediate motor layers of the colliculus, which seems to be much more planar, or at least unfoldable. We will therefore use a Cartesian coordinate system $(X, Y)$ to localize points on the surface of these intermediate or deep layers.

Two-dimensional saccades result from the conjunction of the activity of horizontal and vertical brainstem generators. So the final motor coordinate system is a priori a Cartesian one. However, Robinson Robinson 1972 has shown that for the monkey, the sensorimotor maps of the SC are more adequately described by a deformed polar coordinate system.

The equations mapping retinotopic polar coordinates $(R, \theta)$ onto the collicular surface (Cartesian coordinate $(X, Y)$ in millimeter), first introduced by (Ottes et al. 1986), are:

$$
\begin{aligned}
& X=B_{X} \ln \left(\frac{\sqrt{R^{2}+2 A R \cos (\theta)+A^{2}}}{A}\right) \\
& Y=B_{Y} \operatorname{atan}\left(\frac{R \sin (\theta)}{R \cos (\theta)+A}\right)
\end{aligned}
$$

With the following parameter settings: $A=3.0 \mathrm{deg}$, $B_{X}=1.4 \mathrm{~mm}$ and $B_{Y}=1.8 \mathrm{~mm}$. Even if a precise evaluation of these parameters for the cat was not provided, the cat's mapping depicted in McIlwain 1976) seems to be in accordance with such a description, with a $B_{Y} / B_{X}$ ratio close to 2 .

As noted in (Ottes et al. 1986, this mapping can however be reformulated it as the complex logarithm of a linear function of eccentricity, as proposed by (Schward 1980) in its modeling of the striate cortex mapping. Using $z$, the complex variable defined as:

$$
z=\alpha+i \beta
$$

where $\alpha$ and $\beta$ represent the horizontal and vertical amplitude of the saccade, eqns. 5 and 6 can be rewritten:

$$
\frac{X}{B_{X}}+i \frac{Y}{B_{Y}}=\ln \left(\frac{z+A}{A}\right)
$$

4.2 The need for a linear or complex logarithmic mapping.

The keystone of our result lies in a mathematical formulation of the six biological properties of the spatio-temporal transformation as equations in $\mathbb{C}$.

We work with a complex formulation $S=X+i Y$ of coordinates on the abstract SC map together with a bijection ( $z=\phi(S)$ ) from the colliculus map to the visual hemifield. All along the proof, we will refer to a given desired saccade $z_{0}=\alpha_{0}+i \beta_{0}$ in visual coordinates which can be expressed in collicular coordinates as a specific $S_{0}=\phi^{-1}\left(z_{0}\right)$. The command sent from the superior colliculus to the saccade generators $(\mathrm{H}$ : horizontal, $\mathrm{V}$ : vertical) in order to generate a given $S_{0}$ saccade is described by $O u t_{S_{0}}(t)=O u t_{S_{0}}^{H}(t)+$ $i O u t_{S_{0}}^{V}(t)$. We can now formulate the weighted sum property as

$$
\text { Out }_{S_{0}}(t)=\int_{S} w_{S} \mathcal{A}_{S_{0}}(S, t) d S
$$

where $w_{S} \in \mathbb{C}$ are the weights of connection from the neuron located in $S$ to the saccade generators and $\mathcal{A}_{S_{0}}(S, t) \in \mathbb{R}$ is the activity on the abstract map at location $S$ and time $t$ for a $S_{0}$ saccade. Technically, $\mathcal{A}_{S_{0}}$ is a function such that for all fixed $t$, the product of $\mathcal{A}_{S_{0}}(-, t)$ with any exponential function is of finite integral. For example, it can be a Gaussian or any function with compact support (which will be the case in practice). Similarly, the invariant integral property amounts to say that there exists a function $K_{\mathcal{A}}$ such that

$$
\int_{t} \mathcal{A}_{S_{0}}(S, t) d t=K_{\mathcal{A}}\left(S-S_{0}\right)
$$

and the linearity property expresses that

$$
\int_{t} \text { Out }_{S_{0}}(t) d t=C z_{0} \quad(C \in \mathbb{R})
$$

The similarity property states that for any activation $\mathcal{A}$ that satisfies 10, $w_{S}$ is a similitude in $z$. This is equivalent to the existence of two complex numbers $a$ and $b$ such that

$$
w_{S}=a z+b
$$

Asking for a smooth mapping means that $\phi \in \mathcal{C}^{1}$, satisfies $\phi(0)=0$, and is aligned with the $X$ and $Y$ axes in 0 $\left(D_{X} \phi(0) \in \mathbb{R}^{+}\right.$and $\left.D_{Y} \phi(0) \in i \mathbb{R}^{+}\right)$.

From equations (9), 10) and (11), it is easy to derive:

$$
C \phi\left(S_{0}\right)=\int_{S} w_{S} K_{\mathcal{A}}\left(S-S_{0}\right) d S
$$

We will differentiate this equation with respect to $X$ and $Y$. Let $\psi$ be either $D_{X} \phi$ or $D_{Y} \phi$ and $K=K_{\mathcal{A}}$. Using equation (12) and the fact that $z=\phi(S)$, we get

$$
\forall S_{0} \quad C \psi\left(S_{0}\right)=a \int_{S} K\left(S-S_{0}\right) \psi(S) d S
$$

note that $C, a$ and $K$ depend on $\mathcal{A}$ which is not the case for $\psi$. We now use the possibility to choose different functions for the activity and translate the activity $\mathcal{A}$ for small vectors $u$. We pose $\kappa(\mathcal{A})=C / a$ and introduce the notation $f_{u}(S)=$ $f(S+u)$ for any function $f$

$$
\begin{aligned}
\kappa\left(\mathcal{A}_{u}\right) \psi\left(S_{0}\right) & =\int_{S} K\left(S-\left(u+S_{0}\right)\right) \psi(S) d S \\
& =\int_{S} K\left(S^{\prime}-S_{0}\right) \psi\left(S^{\prime}+u\right) d S \\
& =\kappa(\mathcal{A}) \psi_{u}\left(S_{0}\right)
\end{aligned}
$$

Let $F(u)=\kappa\left(\mathcal{A}_{u}\right) / \kappa(\mathcal{A})$. We have for any small $u$

$$
\psi(S+u)=\psi(S) F(u)
$$

Applying this to $S=0$ leads to $F(u)=\psi(u) / \psi(0)$, so

$$
\psi(S) \psi(u)=\psi(S+u) \psi(0)
$$

Let us introduce the change of coordinates $S \mapsto \widetilde{S}$ that makes the Jacobian of $\widetilde{\phi}$ (ie. the function $\phi$ in the new coordinates) equal to $I$ at 0 . By the hypothesis of smooth mapping, we know that

$$
\widetilde{S}=\frac{X}{b_{X}}+i \frac{Y}{b_{Y}}=\widetilde{X}+i \widetilde{Y}
$$


for some $b_{X}, b_{Y} \in \mathbb{R}^{+}$. By the theorem 1, p 225 of (Bourbaki 1972), we know that $\psi$ is analytic. We then deduce from proposition 7, p 200 of the same book that $\psi$ is an exponential function, ie.

$$
\exists C_{1}, C_{2}, \lambda, \mu \text { in } \mathbb{C}\left\{\begin{array}{l}
D_{X} \widetilde{\phi}(\widetilde{S})=C_{1} \exp (\lambda \widetilde{X}+\mu \widetilde{Y}) \\
D_{Y} \widetilde{\phi}(\widetilde{S})=C_{2} \exp (\lambda \widetilde{X}+\mu \widetilde{Y})
\end{array}\right.
$$

Applying Schwarz's theorem which states that the partial derivatives commute, we infer that $\mu=i \lambda$. To integrate those equalities and obtain the different forms of $\widetilde{\phi}$, we have to distinguish between two cases.

$-\lambda \neq 0$

in that case,

$$
\frac{1}{\lambda}(\exp (\lambda \widetilde{S})-1)=z \quad \lambda \in \mathbb{C}
$$

which can be rewritten if $\lambda \in \mathbb{R}^{+}$

$$
\frac{X}{B_{X}}+i \frac{Y}{B_{Y}}=\ln \left(\frac{z+A}{A}\right)
$$

$-\lambda=0$

in that case,

$$
\widetilde{S}=z
$$

which can be rewritten

$$
\frac{X}{b_{X}}+i \frac{Y}{b_{Y}}=z
$$

Remark that this case is simply the limit case of the exponential mapping when $\lambda \rightarrow 0$.

For our proof to be complete, it remains to check that the necessary conditions found above are also sufficient by explicitly computing $a$ and $b$. To make the formulations simpler, we introduce $u=S-S_{0}$ and $\widetilde{u}=\widetilde{S}-\widetilde{S}_{0}$

$$
\left\{\begin{array}{l}
a=C\left(\sum_{u} \exp (\lambda \widetilde{u}) \cdot K_{\mathcal{A}}(u)\right)^{-1} \\
b=\frac{C}{\lambda}\left(\left(\sum_{u} \exp (\lambda \widetilde{u}) \cdot K_{\mathcal{A}}(u)\right)^{-1}-\left(\sum_{u} K_{\mathcal{A}}(u)\right)^{-1}\right)
\end{array}\right.
$$

\subsection{The gluing of the two colliculi.}

In order to satisfy the Glued colliculi hypothesis of the above proof, we propose a method for gluing the colliculi so that we can then consider them as a single abstract mapping $\phi$ on the whole plane.

We define two distinguished layers Inp and Mot and connect them by direct and commissural connections, as depicted in Fig. 2. The SC neurons sending commissural projections are confined within the boundary of the preferred hemifield, defined by the iso-direction curves $90^{\circ}$ and $-90^{\circ}$ (hatched areas in Fig. 2). By defining the $T$ operator as 1 within this boundary and 0 outside, we can mathematically express $v^{L}$ and $v^{R}$ as follows:

$$
\begin{aligned}
& v^{L}=\sum_{S} T(S) \operatorname{Inp} p^{L}(S, t) \\
& v^{R}=\sum_{S} T(S) \operatorname{Inp} p^{R}(S, t)
\end{aligned}
$$

The sum of the whole activity in one input layer, $\nu$, is defined as:

$$
\nu=\sum_{S} \operatorname{Inp}^{R}(S, t)=\sum_{S} \operatorname{Inp}^{L}(S, t)
$$

We can then relate the four layers by the following equations:

$$
\begin{aligned}
& \operatorname{Mot}^{L}\left(S^{L}, t\right)=f\left(v^{R}\right) \operatorname{Inp} p^{L}\left(S^{L}, t\right) \\
& \operatorname{Mot}^{R}\left(S^{R}, t\right)=f\left(v^{L}\right) \operatorname{Inp} p^{R}\left(S^{R}, t\right)
\end{aligned}
$$

where $S_{0}^{L}$ and $S_{0}^{R}$ are the saccade coordinates expressed in the left and right collicular mappings, $S^{L}$ and $S^{R}$ the coordinates of the considered neuron in the left or right $\mathrm{SC}$, and $f$ is a transfer function tuned to be highly receptive when half of the activity bump enters the boundary of the preferred hemifield. For that, $f$ is a sigmoid with a high steepness $\rho$, centered at one half of $\nu$ :

$$
f(x)=1-\frac{1}{1+\exp ^{a(0.5 \rho-x)}}
$$

The invariant integral property ensures that this four layered structure satisfies relation (4) with $\chi+\eta=1$.

We will call abstract map the result of this gluing.

4.4 Proof of the stability of the similarity under small deformations.

We suppose in this paragraph that the collicular mapping is either logarithmic or linear, and that the collicular output is linear (as required by the linearity hypothesis).

We choose the complex coordinates $S=X+i Y$ on the colliculus and $z$ in the visual field, such that for any $S$, $z=\phi(S)=\exp (S)-1$ or $z=\phi(S)=S$. We have seen that for any given kernel $K_{\mathcal{A}}$ resulting from an invariant integral activity $\mathcal{A}$, integrable with every exponential weights, it exists a similarity $\sigma$ in the $z$-plane, such that for any $S_{0}$ :

$$
\phi\left(S_{0}\right)=\int_{S} K_{\mathcal{A}}\left(S-S_{0}\right) \sigma(\phi(S)) d S .
$$

Suppose that $K_{\mathcal{A}}$ is non identically zero; we want to prove that $\sigma$ is the only function satisfying this equation under natural growth conditions.

Recall the Fourier-Laplace transform of a function (or a distribution) $u$ in the $S$-plane is defined in a point $\zeta=(\xi, \eta)$ in $\mathbb{C}^{2}$, by the integral:

$$
\widehat{u}(\zeta)=\int u(X, Y) e^{-i(\xi X+\eta Y)} d S,
$$


when this integral converges.

We put the hypothesis on $K_{\mathcal{A}}$ that its Fourier-Laplace transform $\widehat{K}$ is defined and complex analytic over the entire complex plane.

Let $f: \mathbb{R}^{2} \rightarrow \mathbb{C}$ be a continuous function, satisfying the equation (26):

$$
\phi\left(S_{0}\right)=\int_{S} K_{\mathcal{A}}\left(S-S_{0}\right) f(S) d S .
$$

Our hypothesis will be that it exists a constant $c$ and and an open set $\Omega$ in $\mathbb{C}^{2}$ containing a plane parallel to $\mathbb{R}^{2}$, such that the difference $\Delta=f-\sigma \circ \phi-c$ has a well defined Fourier-Laplace transform on $\Omega$. This is verified if the gradient $\nabla . \Delta$ is equal to zero for $X$ sufficiently negative or $|Y|$ sufficiently large and is majored by an exponential function for $X$ positive. Remark that the preceding condition means that the deformation $\Delta$ is supported by one of the two colliculi.

Let us denote by $D \Delta$ either $D_{X} \Delta$ or $D_{Y} \Delta$; from equation(26), we have:

$$
\int_{S} K_{\mathcal{A}}\left(S-S_{0}\right) D \Delta(S) d S=0 .
$$

Thus (Hörmander 1983) for $\xi$ in $\Omega$ we obtain:

$$
\widehat{K}(\xi) \widehat{D \Delta}(\xi)=0 .
$$

But when the product of two analytic functions is zero, one of the function is identically zero. As $\widehat{K}$ is not identically zero, $\widehat{D \Delta}$ is zero on $\Omega$, and by the injectivity of the FourierLaplace transform (Hörmander 1983), $D \Delta$ itself is zero. Thus $f$ is a similarity. Q.E.D.

Remarks:

1. if $K_{\mathcal{A}}$ and $D \Delta$ were continuous functions (or even distributions) with compact support (which is not so restrictive when considering neural activity on SC maps), we could have directly deduced the result $D \Delta=0$ from the classical "Theorem of Supports" of Titchmarsh and Lions (Hörmander 1983).

2. it is not true in general that equation (26) has a unique solution, for example if the total integral of $K$ is zero we can add any constant to $\sigma$; moreover if the Fourier transform of $K$ becomes zero at some points in $\mathbb{R}^{2}$ it exists non trivial polynomial function $\Delta$ verifying (29), their Fourier transform having support reduced to isolated points. This phenomenon cannot appear when $K$ belongs to the class of "Wiener functions" which by definition have Fourier transforms without zero, in this case $\Delta$ can be any tempered distribution in the sense of Schwartz and we deduce $D \Delta=0$.

In the special case of Gaussian integral of activities $K_{\mathcal{A}}$, the restrictive hypothesis on $\Delta$ can be greatly weakened: we only have to require that it exists two real constants $\alpha, \beta$ such that $\Delta$ has a well defined Fourier-Laplace transform, as an element of the space $\mathcal{S}^{\prime}$ of Schwartz tempered distributions, on the plane $\Pi=(i \alpha+\xi, i \beta+\eta) \mid(\xi, \eta) \in \mathbb{R}^{2}$ in $\mathbb{C}^{2}$.
For example $\Delta$ can be any function with polynomial growth times an exponential. Let us prove that this condition is sufficient to imply $D \Delta=0$ :

By hypothesis it exists a positive symmetric two by two matrix of determinant one $A$, a point $M_{O}$ in $\mathbb{R}^{2}$, a constant $C>0$ and a number $\tau>0$, such that

$$
K(S)=C(4 \pi \tau)^{-1} e^{-\left(S-M_{0}\right) \cdot A\left(S-M_{0}\right) / 4 \tau} .
$$

The Fourier-Laplace transform of $K$ is the analytic function

$$
\widehat{K}(\zeta)=C e^{-\tau \zeta \cdot A^{-1}(\zeta)+i \zeta \cdot M_{0}}
$$

On the other hand $D \Delta$ has a Fourier-Laplace transform, well defined as a tempered distribution on the plane $\Pi$, it is the product of $\widehat{\Delta}$ with the restriction to $\Pi$ of a linear form on $\mathbb{C}^{2}$. The convolution equation satisfied by $D \Delta$ implies as before $\hat{K} \widehat{D \Delta}=0$, but $\widehat{K}$ restricted to $\Pi$ belongs to the test space $\mathcal{S}$ of Schwartz functions with quick decreasing at infinity, and has no zero at all, so $\widehat{D \Delta}=0$. Q.E.D.

\subsection{Proof of the need for an invariant integral.}

We now replace the invariant integral property by the fact that any logarithmic mapping works. We show that equation 10 can be deduced, ie.

$$
\int_{t} \mathcal{A}_{S_{0}}(S, t) d t=K_{\mathcal{A}}\left(S, S_{0}\right)=K_{\mathcal{A}}\left(S-S_{0}, 0\right)
$$

Using equations 9, 11 and 12 for any logarithmic mapping leads to

$$
\forall \lambda \quad C e^{\lambda S_{0}}=\int_{S}\left(a e^{\lambda S}+b\right) K_{\mathcal{A}}\left(S, S_{0}\right) d S
$$

We differentiate with respect to $\lambda$

$$
\forall \lambda \quad \kappa(\mathcal{A}) e^{\lambda S_{0}}=\int_{S} e^{\lambda S} K_{\mathcal{A}}\left(S, S_{0}\right) d S
$$

We pose $\Delta\left(S, S_{0}\right)=K_{\mathcal{A}}\left(S, S_{0}\right)-K_{\mathcal{A}}\left(S-S_{0}, 0\right)$ and deduce that

$$
\forall \lambda \quad \int_{S} e^{\lambda\left(S-S_{0}\right)} \Delta\left(S, S_{0}\right) d S=0
$$

The Laplace transform is defined for $\mathcal{A}$, thus it is also defined for $K_{\mathcal{A}}=\int_{t} \mathcal{A} d t$, as the integration is on a finite interval, and finally for any difference of to such $K$ functions, in particular for $\Delta$. Then, with the same argument as in section 4.4, we have that

$$
\Delta\left(S, S_{0}\right)=0
$$


4.6 A generalization of the similarity hypothesis.

If we relax the hypothesis of similarity by just asking for an affine projection, ie. $w_{S}=\mathbf{a} z+b$ where $\mathbf{a}$ is a $2 \times 2$ invertible matrix, we then get five types of solutions.

Indeed, denoting by $J(S)$ the Jacobian of $\phi$ at point $S$ leads to

$$
J(u) J(0)^{-1} J\left(S_{0}\right)=J\left(u+S_{0}\right)
$$

As above, we perform the change of coordinates $S \mapsto \widetilde{S}$ to make $J(0)=I$. As in section 4.2, using theorem 1, p 225 and proposition 7, p 200 of (Bourbaki 1972) guarantees the existence of two commuting matrices $M_{1}$ and $M_{2}$ such that

$$
\widetilde{J}(\widetilde{S})=\exp \left(M_{1} X+M_{2} Y\right)
$$

By distinguishing between the different kind of sub-vector spaces $\mathbb{R}\left(M_{1}, M_{2}\right)$, we obtain five solutions, where $P$ is a $2 \times 2$ invertible matrix (allowing a twist in the mapping) and $W=P^{-1}(\widetilde{S})$ is seen as a complex number $U+i V$.

1. $z=P \frac{1}{\lambda}(\exp (\lambda W)-1) \quad \lambda \in \mathbb{C}$

2. $z=P\left(\begin{array}{c}\frac{1}{\lambda}(\exp (\lambda U)-1) \\ \frac{1}{\mu}(\exp (\mu V)-1)\end{array}\right) \quad \lambda, \mu \in \mathbb{R}$

3. $z=P\left(\begin{array}{c}\frac{1}{\lambda}(\exp (\lambda U)-1) \\ \exp (\lambda U)\left(V+\frac{\nu}{\lambda}\left(\frac{1}{\lambda}-U\right)\right)-\frac{\nu}{\lambda^{2}}\end{array}\right) \quad \lambda, \nu \in \mathbb{R}$

4. $z=P\left(\frac{\frac{1}{\lambda}(\exp (\lambda U)-1)}{V}\right) \quad \lambda \in \mathbb{R}$

5. $z=\widetilde{S}$

Some examples of these mappings, with and without deformations are depicted in Fig. 6 .

\subsection{Description of the gluing simulation.}

Collicular maps are modeled by $90 \times 90$ tables of leakyintegrator neurons including 15 neurons borders. The system has the following hierarchical structure (see also Fig. 可):

1. The retina Ret encodes the target's position by a $2 \mathrm{D}$ Gaussian with standard deviation $\sigma=2.5$, centered around target's position.

2. The input layers Inp receives the retinal signal with $70 \mathrm{~ms}$ delay. When the global activity passes a given threshold, it is transmitted to the motor layers (via a gluing mechanism which implements either ours or the Van Gisbergen's scheme) and the SBG OPNs are inhibited via LLBs.

3. The motor layers Mot send the command to the SBG, while their activities are integrated. When the integrator reaches a given threshold, the layers are inhibited and the saccade stops.

4. The SBG is first inhibited by the OPNs. The activity in Inp is transmitted to the LLBs which inhibit in turn the OPNs. When the activity in Inp is strong enough, OPNs are turned off and the EBNs/IBNs begin to receive the motor command from the Mot layers through a weighted sum. This command is then integrated by the

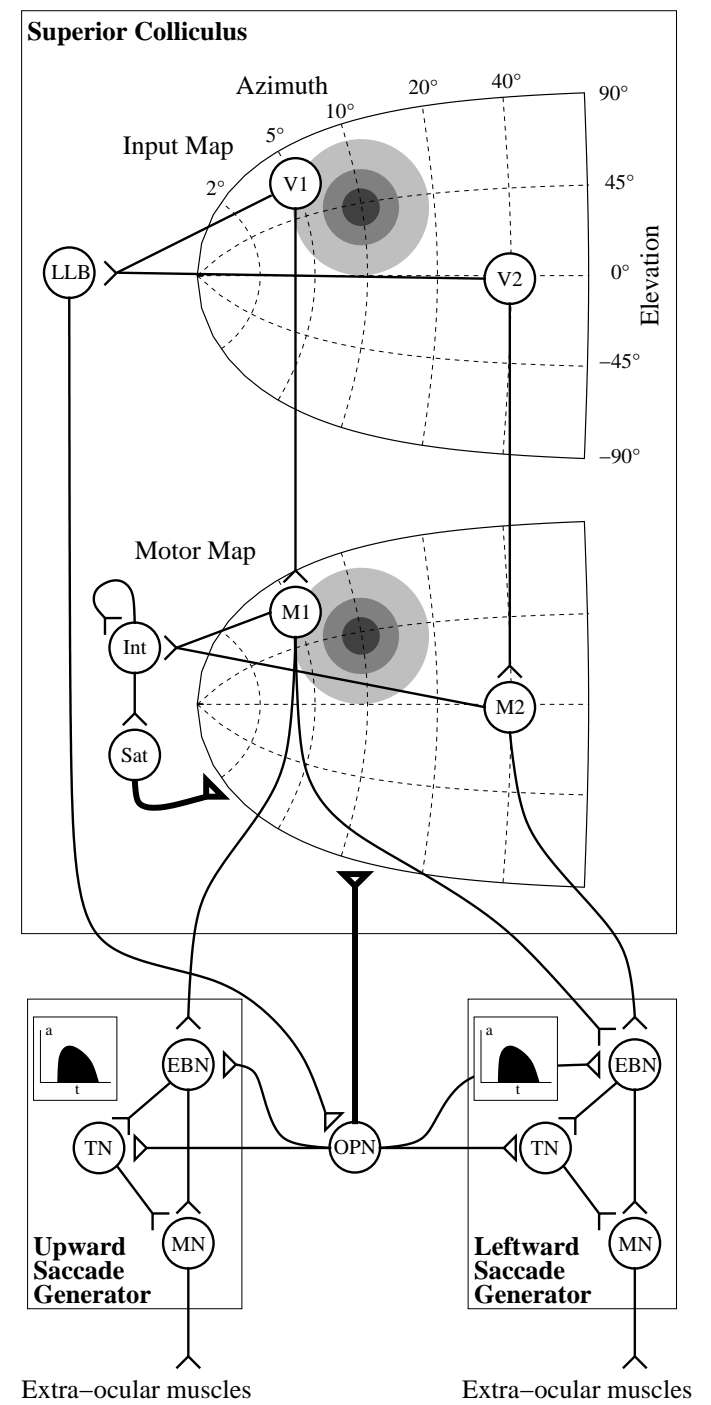

Fig. 7 Gluing simulation architecture. For simplicity, only one colliculus and two SBG (upward and leftward), without the crossed IBN projections, are represented. Moreover, only two neurons are represented in each collicular map (V1, V2 and M1, M2, for visual and motor maps respectively). Shaded circles in collicular maps represent the Gaussian activity generated by a $\left(10^{\circ}, 10^{\circ}\right)$ target, while insets in the saccade generators represent the temporal code in the EBNs generated to drive the muscles. Open triangles represent excitatory synapses; triangles represent inhibitory synapses; bold connections affect the whole map. Refer to text for the abbreviations.

couple of neurons TNs/MNs (tonic neurons/motoneurons). The activity of MNs is received by the eye plant (modeled by a second order differential equation) to generate the required eye's displacement.

The leaky-integrator rate neuron model used as building brick is as follows $(\tau$ : time constant in $m s, I$ : input in $m V)$ :

$$
\tau \frac{d a}{d t}=I-a \text { and } y=[a]^{+}
$$

where the transfer function []$^{+}$satisfies $[I]^{+}=0$ if $I<0$ and $[I]^{+}=I$ otherwise. 

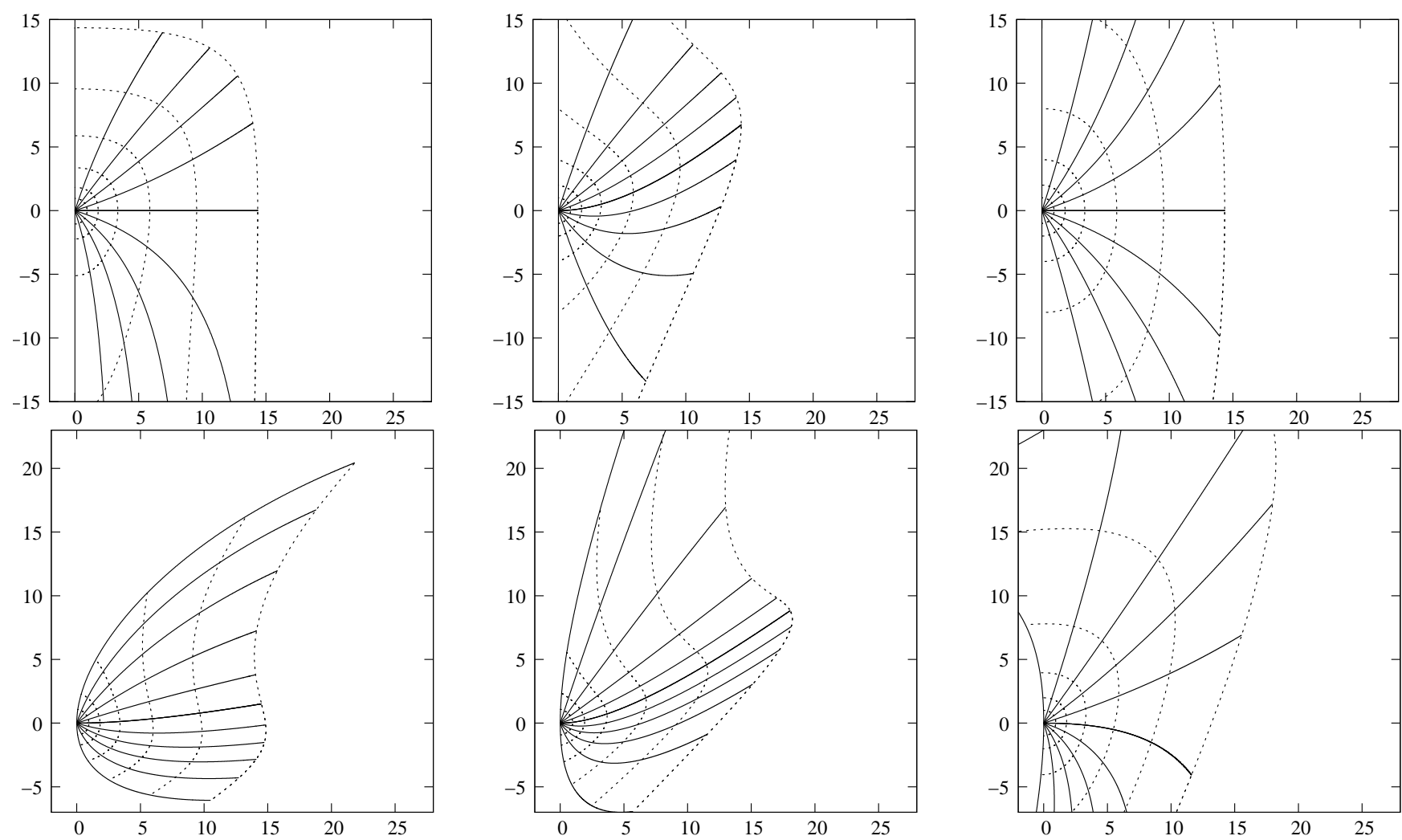

Fig. 6 Examples of the mappings predicted by a relaxation of the similarity hypothesis. The top row contains the new mappings defined in section 4.6 without deformation (P is the identity matrix): from left to right, mapping 2 . $(\lambda=0.1$ and $\mu=0.1)$, mapping 3 . ( $\lambda=0.1$ and $\nu=0.1)$, and mapping 4. $(\lambda=0.1)$. The bottom row represents mapping 1 . (complex logarithmic, $\lambda=0.1)$, mapping 3 . $(\lambda=0.1$ and $\nu=0.1)$ and mapping $4(\lambda=0.1)$ with the deformation matrix $P=[1,0.5 ; 0.2,1]$. The dashed lines represent iso-amplitudes and full lines, iso-directions, as in Fig. 1. The axes units are millimeters and the same $B_{x}=1.4 \mathrm{~mm}$ and $B_{y}=1.8 \mathrm{~mm}$ parameters are used for all maps.

The input of $\operatorname{Inp}$ is:

$$
I_{\text {Inp }}\left(S_{0}^{D}, t\right)=y_{\operatorname{Ret}^{D}}\left(S_{0}, t-t_{0}\right) \text {, with } D \in\{L, R\}
$$

Long-Lead burst neurons (LLB), in charge of triggering saccades by inhibiting the OPN when the activity in the Input layers reaches the $\epsilon_{\text {trig }}$ threshold, are modeled by:

$$
\begin{gathered}
I_{L L B}=w_{V i s}^{L L B} \sum_{S}\left(y_{I n p^{R}}(S)+y_{I n p^{L}}(S)\right)-\epsilon_{t r i g} \\
I_{O P N}=-y_{L L B}+\epsilon_{O P N}
\end{gathered}
$$

The activity in the motor layer $M$ ot is gated by the OPNs and the Integrating-Saturating mechanism (note that saturation neurons have a longer time constant):

$$
I_{M o t} D(S)=y_{I n p^{D}}(S)-w_{O P N}^{M o t} y_{O P N}-w_{\text {Sat }}^{M o t} y_{S a t}
$$

with $D \in\{R, L\}$.

$$
\begin{gathered}
I_{\text {Int }}=w_{M o t}^{\text {Int }} \sum_{S}\left(y_{M o t^{R}}(S)+y_{M o t^{L}}(S)\right) \\
I_{\text {Sat }}=y_{\text {Int }}-\epsilon_{\text {stop }}
\end{gathered}
$$

The four SBG circuits (leftward, rightward, upward, downward) are identical, all of them are gated by OPN activity, and those operating in opposite directions are coordinated by the IBN crossed projections. The EBN and IBN activity is identical and defined by:

$$
\begin{aligned}
& I_{B N}^{D}=\sum_{X, Y}\left(w_{\alpha} y_{M o t}(S)\right)-w_{O P N}^{B N} y_{O P N}, \text { for } D \in\{L, R\} \\
& I_{B N}^{D}=\sum_{X, Y}\left(w_{\beta} y_{M o t}(S)\right)-w_{O P N}^{B N} y_{O P N}, \text { for } D \in\{U, D\}
\end{aligned}
$$

with the $w_{\alpha}$ and $w_{\beta}$ defined in equation (3) of the main manuscript.

The tonic neurons are the only neurons modeled as perfect rather than leaky-integrators:

$$
I_{T N}^{D}=w_{B N}^{T N}\left(y_{E B N}^{D}-y_{I B N}^{D^{o p}}\right) \text {, with } D \in\{U, D, L, R\}
$$

$I_{M N}^{D}=w_{B N}^{M N}\left(y_{E B N}^{D}-y_{I B N}^{D^{o p}}\right)+y_{T N}$, with $D \in\{U, D, L, R\}$ where $D^{o p}$ is the opposite direction of $D$.

The eye plant model used is modeled as a second order differential equation:

$$
\ddot{\theta}+0.6 \dot{\theta}+4 \theta=w_{M N}^{\theta} y_{M N}
$$

The parameters are summed up in the Table 1. 
Table 1 Parameters of the model

\begin{tabular}{c|cc|cc|c}
$\tau$ & $5 m s$ & $\tau_{\text {Sat }}$ & $100 m s$ & $t_{0}$ & $70 m s$ \\
$\epsilon_{O P N}$ & 100 & $\epsilon_{\text {trig }}$ & 400 & $\epsilon_{\text {stop }}$ & 200 \\
$w_{V i s}^{L L B}$ & 0.005 & $w_{O P N}^{M o t}$ & 40 & $w_{O P N}^{B N}$ & 40 \\
$w_{M o t}^{\text {Int }}$ & 0.002 & $w_{\text {Sat }}^{\text {Mot }}$ & 8 & $w_{B N}^{T N}$ & 0.05 \\
$w_{B N}^{M N}$ & 1.52 & $w_{M N}^{\theta}$ & 4.07 &
\end{tabular}

Acknowledgements The authors would like to gratefully thank A Grantyn and A. Moschovakis for the valuable discussions, and H. Hicheur for valuable suggestions concerning the manuscript.

\section{References}

Anderson, R., Keller, E., Gandhi, N., and Das, S. (1998). Twodimensional saccade-related population activity in superior colliculus in monkey. J Neurphysiol, 80(2):798-817.

Arai, K., Das, S., Keller, E., and Aiyoshi, E. (1999). A distributed model of the saccade system: simulations of temporally perturbed saccades using position and velocity feedback. Neural Netw, 12(10):1359-1375.

Arai, K., Keller, E., and Edelman, J. (1994). Two-dimensional neural network model of the primate saccadic system. Neural Netw, 7:1115-1135.

Badler, J. and Keller, E. (2002). Decoding of a motor command vector from distributed activity in superior colliculus. Biol Cybern, 86(3):179-189.

Bourbaki, N. (1972). Groupes et Algèbres de Lie, Chapitres 2 et 3. Dunod, Paris, France.

Dräger, U. and Hugel, D. (1976). Topography of visual and somatosensory projections to mouse superior colliculus. J Neurophysiol, 39:91-101.

Feldon, S., Feldon, P., and Kruger, L. (1970). Topography of the retinal projection upon the superior colliculus of the cat. Vision Res, 10:135-143.

Girard, B. and Berthoz, A. (2005). From brainstem to cortex: Computational models of saccade generation circuitry. Progress in Neurobiology, 77:215-251.

Goffart, L. and Pélisson, D. (1998). Orienting gaze shifts during muscimol inactivation of caudal fastigial nucleus in the cat. i. gaze dysmetria. J. Neurophysiol., 79:1942-1958.

Goossens, H. and van Opstal, A. (2000). Blink-perturbed saccades in monkey. II. superior colliculus activity. Journal of Neurophysiology, 83:3430-3452.

Goossens, H. and van Opstal, A. (2006). Dynamic ensemble coding of saccades in the monkey superior colliculus. J. Neurophy., 95:2326-2341.

Grantyn, A., Brandi, A.-M., Dubayle, D., Graf, W., Ugolini, G., Hadjidimitrakis, K., and Moschovakis, A. (2002). Density gradients of trans-synaptically labeled collicular neurons after injections of rabbies virus in the lateral rectus muscle of the rhesus monkey. $J$ Comp Neurol, 451:346-361.

Grantyn, A. and Moschovakis, A. (2003). Structure-function relationships in the superior colliculus of higher mammals. In Hall, W. and Moschovakis, A., editors, The superior colliculus: new approaches for studying sensorimotor integration, Methods \& new frontiers in neuroscience, chapter 5, pages 107-145. CRC Press, Boca Raton, FL.

Groh, J. (2001). Converting neural signals from place codes to rate codes. Biol. Cybern., 85(3):159-165.
Herrero, L., Rodríguez, F., Salas, C., and Torres, B. (1998). Tail and eye movememnts evoked by electrical microstimulation of the optic tectum in goldfish. Exp Brain Res, 120:291-305.

Hirsch, H. (1976). Differential topology. Springer-Verlag, New York.

Hörmander, L. (1983). The Analysis of Linear Partial Differential Operators I. Number 256 in Grundlehren der mathematischen Wissenschaften. Springer-Verlag, Berlin, Heidelberg, New-York, Tokyo.

Iwamoto, Y. and Yoshida, K. (2002). Saccadic dysmetria following inactivation of the primate fastigial oculomotor region. Neurosci. Lett., 325:211-215.

Kaneko, C., Evinger, C., and Fuchs, A. (1981). Role of the cat pontine burst neurons in generation of saccadic eye movements. J Neurophysiol, 46(3):387-408.

Keller, E. (1974). Participation of medial pontine reticular formation in eye movement generation in monkey. J Neurophysiol, 37(2):316332.

King, W. and Fuchs, F. (1979). Reticular control of vertical saccadic eye movements by mesencephalic burst neurons. J Neurophysiol, 42(3):861-876.

Knudsen, E. (1982). Auditory and visual maps of space in the optic tectum of the owl. J Neurosci, 2(9):1177-1194.

Lee, C., Rohrer, W., and Sparks, D. (1988). Population coding of saccadic eye movements by neurons in the superior colliculus. $\mathrm{Na}$ ture, 332:357-360.

McIlwain, J. (1976). Large receptive fields and spatial transformations in the visual system. In Porter, R., editor, Neurophysiology II, volume 10 of Int Rev Physiol, pages 223-248. University Park Press, Baltimore.

McIlwain, J. (1983). Representation of the visual streak in visuotopic maps of the cat's superior colliculus: influence of the mapping variable. Vision Res, 23(5):507-516.

Moschovakis, A., Kitama, T., Dalezios, Y., Petit, J., Brandi, A., and Grantyn, A. (1998). An anatomical substrate for the spatiotemporal transformation. J Neurosci, 18(23):10219-10229.

Munoz, D., Waitzman, D., and Wurtz, R. (1996). Activity of neurons in monkey superior colliculus during interrupted saccades. Journal of Neurphysiology, 75(6):2562-2580.

Olivier, E., Porter, J., and May, P. (1998). Comparison of the distribution and somatodendritic morphology of tectotecal neurons in the cat and monkey. Vis. Neurosci., 15:903-922.

Optican, L. (2005). Sensorimotor transformation for visually guided saccades. Annals of the New York Academy of Sciences, 1039:132148.

Ottes, F., van Gisbergen J.A., and Eggermont, J. (1986). Visuomotor fields of the superior colliculus: a quantitative model. Vision Res, 26(6):857-873.

Robinson, D. (1972). Eye movements evoked by collicular stimulation in the alert monkey. Vision Res, 12:1795-1808.

Rosa, M. and Schmid, L. (1994). Topography and extent of visual-field representation in the superior colliculus of the megachiropteran Pteropus. Vis Neurosci, 11:1037-1057.

Schwarz, E. (1980). Computational anatomy and functional architecture of striate cortex: A spatial mapping approach to perceptual coding. Vision Res, 20:645-669.

Siminoff, R., Schwassmann, H., and Kruger, L. (1966). An electrophysiological study of the visual projection to the superior colliculus of the rat. J Comp Neurol, 127:435-444.

Soetedjo, R., Kaneko, C., and Fuchs, A. (2000). Evidence that the superior colliculus participates in the feedback control of saccadic eye movements. Journal of Neurophysiology, 87:679-695.

Sparks, D., Holland, R., and Guthrie, B. (1976). Size and distribution of movement fields in the monkey superior colliculus. Brain Res, 113:21-34.

van Gisbergen, J., van Opstal, A., and Tax, A. (1987). Collicular ensemble coding of saccades based on vector summation. Neuroscience, 21(2):541-555.

van Opstal, A. and van Gisbergen, J. (1989). A nonlinear model for collicular spatial interactions underlying the metrical properties of electrically elicited saccades. Biol Cybern, 60(3):171-183. 
Yoshida, K., McCrea, R., Berthoz, A., and Vidal, P. (1982). Morphological and physiological characteristics of inhibitory burst neurons controlling horizontal rapid eye movements in the alert cat. $J$ Neurophysiol, 48(3):761-784. 